\title{
Outcome of antenatally detected cystic dysplastic kidney disease
}

\author{
N Al-Khaldi, A R Watson, J Zuccollo, P Twining, D H Rose
}

\begin{abstract}
Forty four fetuses with multicystic dysplastic kidney (MCDK) disease recognised on antenatal ultrasound were studied prospectively. In nine aborted fetuses and in five who died in the neonatal period the MCDK disease was bilateral or there were associated lethal abnormalities or syndromes. All surviving infants had unilateral disease and in six $(20 \%)$ there was significant reflux into the normal contralateral kidney. Since 1988 the management of unilateral MCDK disease has been conservative with no child developing sepsis, hypertension, or malignancy. Serial ultrasound examinations suggest that MCDK lesions involute with time and conservative rather than operative management is favoured.

(Arch Dis Child 1994; 70: 520-522)
\end{abstract}

Cystic lesions affecting the kidney are being detected more often with the widespread use and better resolution of antenatal ultrasound. ${ }^{1}$ The most common cystic lesion recognised antenatally is multicystic dysplastic kidney (MCDK) disease. In this disorder renal dysplasia is associated with a variable number of cysts and is believed to result from failed coordination and development of the metanephros and the branching ureteric bud. ${ }^{2}$ ${ }^{3}$ Most cases are unilateral and MCDK disease is the second most common cause of an antenatally detected urinary tract abnormality. ${ }^{14}$

Dysplastic kidney disease may be bilateral in association with syndromal and nonsyndromal disorders. Cysts may also develop in areas of renal dysplasia associated with urinary tract obstruction. ${ }^{2}$ It is important that cystic dysplastic kidney disease is distinguished from autosomal recessive or dominant polycystic kidney disease for prognosis and counselling.

Since 1985 we have maintained a registry of antenatally detected urinary tract abnormalities in collaboration with colleagues in obstetrics, radiology, pathology, and genetics. A perinatal discussion group meets monthly to review antenatally detected problems and outcome. Liveborn infants have been carefully investigated using a standard protocol.

We report our experience with antenatally detected cystic dysplastic renal disease to emphasise the poor prognosis with bilateral disease and the relatively benign outcome with unilateral lesions. The latter may be associated with significant abnormalities in the contralateral kidney, however, and controversy still exists between conservative or operative management of unilateral MCDK disease. ${ }^{5}$

\section{Methods}

Details were obtained from the registry of all cases of cystic dysplastic kidney disease recognised antenatally from 1985 to 1992 . This included cases where termination or spontaneous abortion took place and a necropsy was performed. Only those fetuses from pregnancies booked at either the City or University Hospitals, Nottingham were included when calculating the incidence of MCDK disease.

The policy for obstetric ultrasound changed during the duration of the study. The initial booking scan at 12 to 16 weeks has been replaced by a detailed scan at 18 weeks in the University Hospital and largely replaced by 18 week detailed scanning at the City Hospital. Antenatal counselling by a paediatric nephrologist (ARW) has been given when requested by the obstetrics staff.

Examination of the liveborn infants included careful attention to palpable masses and any other congenital abnormalities. An ultrasound scan was usually performed after the first day of life and a micturating cystourethrogram with two days of trimethoprim antibiotic cover within the first month. A dimercaptosuccinic acid (DMSA) scan, urine culture, and determination of plasma creatinine and electrolytes were carried out within in the first three months after birth as a day case procedure. No child has required an intravenous urogram. Follow up ultrasound examinations were initially performed at 1 year but subsequently have been deferred to 2 and 5 years of age.

Two of the 30 liveborn infants underwent nephrectomy but since 1987 no child has been referred for an operation. All patients are seen routinely at six monthly intervals when growth is assessed and blood pressure and urine cultures obtained. Prophylactic antibiotics with trimethoprim ( $2 \mathrm{mg} / \mathrm{kg}$ body weight) as a single evening dose were used only when vesicoureteric reflux had been shown on the initial micturating cystourethrogram.

\section{Results}

Forty four cases of MCDK disease were recorded in the seven year period. Twenty $(45 \%)$ of MCDK lesions were detected in the second trimester. Twenty seven $(61 \%)$ were in male fetuses.

Eight pregnancies were terminated between 15 and 25 weeks' gestation, and there was one spontaneous abortion at 15 weeks. All aborted pregnancies had either bilateral MCDK disease or in one instance contralateral agenesis of the kidney (table). In five of the nine fetuses there was associated lower urinary tract obstruction and an oligohydramnios 
Antenatally detected MCDK disease: associated abnormalities

\begin{tabular}{ll}
\hline Abnormality & $\begin{array}{l}\text { No of } \\
\text { cases }\end{array}$ \\
\hline $\begin{array}{l}\text { Aborted fetuses (n=9) } \\
\text { Lower urinary tract obstruction }\end{array}$ & 3 \\
$\begin{array}{c}\text { Anorectal atresia } \pm \text { vaginal atresia } \pm \text { congenital heart } \\
\text { lesion }\end{array}$ & 3 \\
$\begin{array}{l}\text { Fallot's tetralogy and absent right kidney } \\
\text { Meckel-Gruber syndrome }\end{array}$ & 1 \\
Dandy-Walker syndrome & 1 \\
$\begin{array}{l}\text { Neonatal deaths (n=5) } \\
\text { Bilateral MCDK disease with lung hypoplasia }\end{array}$ & 1 \\
Trisomy 18 (single MCDK disease) & 2 \\
Chromosomal deletion (45X/46Xq26 single MCDK & 1 \\
$\quad$ disease) & 1 \\
Hypoplastic left heart with single MCDK disease & 1 \\
Vesicoureteric reflux into contralateral kidney & 6 \\
Vesicoureteric reflux into ureter of cystic kidney & 4 \\
Pelviureteric junction obstruction in contralateral kid- & \\
ney & 1 \\
\hline
\end{tabular}

sequence pattern at necropsy. Five infants died in the neonatal period. Again the cystic kidney disease was either bilateral or associated with other abnormalities (table). The incidence of antenatally detected unilateral MCDK disease in Nottingham was 1 in 3100 total births over a four year period 1988-91 (14 cases and 43 419 total births)

The 30 surviving liveborn infants all had an unilateral MCDK lesion which was left sided in 15 . Eleven $(37 \%)$ had a palpable kidney at birth. All underwent ultrasonography, micturating cystourethrography, and a DMSA scan. The ultrasound scan confirmed the diagnosis of unilateral MCDK disease in all cases and suggested contralateral pelviureteric junction obstruction in one infant. Vesicoureteric reflux into the contralateral ureter was found in six $(20 \%)$ children. In all instances the reflux was moderate (grade II) with no associated dilatation on the ultrasound scan. In four infants there was also reflux into the atretric ureter on the side of the cystic kidney.

The patients have been followed up for a mean of 2.4 years (range 0.2 to 7 years). No child has developed hypertension and urinary infections have not occurred in patients with or without vesicoureteric reflux. All children maintained normal growth centiles except two infants where social and constitutional factors were felt to be operative. A two year follow up ultrasound scan has been completed in 18 children and eight showed involution or a decrease in size.

\section{Discussion}

The present study emphasises that bilateral cystic dysplastic kidney disease is almost invariably associated with a poor outcome because oligohydramnios and other congenital abnormalities predominate. The apparent high incidence of bilateral MCDK disease in this series $(10 / 44(23 \%)$ fetuses) may be due to the prospective data collection and the close collaboration of a perinatal pathologist. Cystic dysplastic lesions found in these multisystem disorders are part of the syndrome, and cystic renal dysplasia may also occur in congenitally obstructed kidneys. ${ }^{2}$ It is important to distinguish MCDK disease from autosomal dominant or recessive polycystic kidney disease as there are obvious implications for prognosis and genetic counselling.

When MCDK disease is unilateral it is usually an asymptomatic disorder with a good prognosis. Although $37 \%$ of the liveborn infants in our series had a palpable kidney at birth this figure was influenced by the paediatric staff being forewarned. Multicystic dysplastic kidney disease is quoted as being one of the most common causes of a unilateral renal mass in the neonatal period. ${ }^{6}$

Previous studies have drawn attention to abnormalities in the contralateral normal kidney with rates as high as $20-80 \%$. $^{6-8}$ These studies have included both prenatally diagnosed MCDK disease and those detected later in infancy, however. This is the first study where infants with antenatally detected pathology have been investigated in a systematic manner. It confirms that there is a significant percentage of abnormalities to be found in the contralateral kidney, particularly vesicoureteric reflux ( $20 \%$ in our series). This obviously has important implications for assessing the long term outcome in these children with only one normal single kidney. We suggest that a micturition cystourethrogram should be part of the routine initial investigations in this disorder. Others have commented on the high rate of urinary infection in their children, but this has not been our experience with the children maintained on prophylactic trimethoprim ( $2 \mathrm{mg} / \mathrm{kg} / \mathrm{day})$ for the first two years of life.

There are reports of multicystic dysplastic kidneys being associated with sepsis, hypertension, or even malignancy. ${ }^{9-10}$ Further experience and more detailed studies suggest that the risk of such complications is low. ${ }^{11} 12$ None has been noted in our series to date and as many courses of unilateral MCDK disease appear to involute with time a conservative approach appears to be justified. ${ }^{13-16}$ Indeed, had the MCDK lesion not been identified prenatally many of these infants would have been regarded as having unilateral renal agenesis. Further follow up is required to fully define the national history of this disorder.

We thank Annette Frogson for typing the manuscript, and those who contributed to the registry.

1 Watson AR, Readett D, Nelson CS, Kapila L, Mayell MJ. Dilemmas associated with antenatally detected urinary Dilemmas associated with antenatally detected urinar tract abnormalities. Arch Dis Child 1988; 63: 719-22.
Kissane JM. Renal cysts in paediatric patients: a classifiKissane JM. Renal cysts in paediatric patients: a classit
cation and overview. Pediatr Nephrol 1990; 4: 69-77.

3 Bernstein J. A classification of renal cysts. In: Gardner KD $\mathrm{Jr}$, ed. Cystic diseases of the kidney. New York: Wiley, 1976 $7-30$.

4 Scott JES, Renwick $M$. Urological anomalies in the Northern Region Fetal Abnormality Survey. Arch Dis Child 1993; 68: 22-6.

5 Hartman GE, Smolik LM, Shochat SJ. The dilemma of the multicystic dysplastic kidney. $A m \mathcal{F}$ Dis Child 1986; 140: 925-8.

6 Atiyeh B, Husmann D, Baum M. Contralateral renal abnormalities in multicystic-dysplastic kidney disease. $\mathcal{f}$ Pediat 1992; 121: 65-7.

7 Walker D, Fennel R, Giorin E, Richard G. Spectrum of multicystic renal dysplasia: diagnosis and management. Urology 1978; 11: 433-6.

8 Kleiner B, Filly RA, Mack L, Callen PW. Multicystic dysplastic kidney: observations of contralateral disease in the fetal population. Radiology 1986; 161: 27-9.

9 Birken G, Kind D, Vane D, Lloyd T. Renal cell carcinoma arising in a multicystic dysplastic kidney. $\mathcal{f}$ Pediatr Surg $1985 ; 20: 619$. 
10 Chen YH, Stapleton FB, Roy S III, Noe HN. Neonata hypertension from a unilateral multicystic dysplastic hypertension from a unilateral
kidney. $f$ Urol 1985; 133: 664 .

11 Dimmick JE, Johnson HW, Coleman GU, Carter M. Wilms tumor, nodular renal blastema and multicystic renal dystumor, nodular renal blastema

12 Gordan AC, Thomas DFM, Arthur RJ, Irving HC Multicystic dysplastic kidney: is nephrectomy stil appropriate? F Urol 1988; 140: 1231-4.

13 Pedicelli G, Jequier S, Bowen AD, Boisvert J. Multicystic dysplastic kidneys: spontaneous regression demonstrated with US. Radiology 1986; 161: 23-6.
14 Avni EF, Thoua Y, Lalmand B, Didier F, Droulle P Schulman CC. Multicystic dysplastic kidney: natural history from in utero diagnosis and postnatal follow up. $f$ hrol 1987; 138: 1420-4.

15 Vinocur L, Slovis TL, Perlmutter AD, Watts FB, Chang $\mathrm{CH}$. Follow-up studies of multicystic dysplastic kidneys. Radiology 1988; 167: 311-5.

16 Rickwood AMK, Anderson PAM, Williams MPL Multicystic renal dysplasia detected by prenatal ultrasonography. Natural history and results of conservative management. Br $\mathcal{F}$ Urol 1992; 69: 538-40.

\section{Eye poking - comme avec un tisonnier}

Some rub their eyes, some press them, and some poke them. These behaviours have been lumped together as 'oculodigital phenomena' but workers in Vancouver (James E Jan and colleagues, Developmental Medicine and Child Neurology 1994; 36: 321-5) have pointed out that their associations are different. Eye rubbing is normal; it is a commonplace observation that most children and many adults rub their eyes when they are tired. Eye pressing occurs in children with severe bilateral congenital visual impairment due to disease of the eyes often when they are bored or anxious and sometimes while listening to music. Children with cortical blindness do not do it. (Some paediatric neurologists have been known to do it but not, so far as is generally known, to themselves.) It is not harmful. Eye poking, though, is a different matter, being very definitely harmful. Here the child pokes painfully at the eye, usually with the ipsilateral forefinger. Among 500 highly selected children referred to the Visually Impaired Programme of the Children's Hospital in Vancouver, 21 were given to eye poking. All 21 suffered from multiple handicapping conditions with severe global learning disorder. Fifteen of them were visually impaired but that was thought to represent ascertainment bias and there was no correlation between the degree of visual loss and the severity of eye poking. There were often other self injurious behaviours such as head banging, hand biting, hair pulling, and face slapping. Eye poking was more common in boys (13 boys, eight girls) but, strangely, the authors do not mention Lesch-Nyhan syndrome. The behaviour led to enucleation of an eye in one child and corneal scarring in three others. Retinal detachment, cataracts, ocular infection, and intraocular bleeding have all been described. These authors give a list of drugs which have been suggested and recommend behaviour modification but no treatment seems to have been successful.

Developmental Medicine and Child Neurology provides French, German, and Spanish translations of its summaries. I enjoyed the French translation of eye poking; 'se fourrer le doigt sur l'oeil, comme avec un tisonnier' (to stick your finger in your eye, as with a poker). Quite so! 